\title{
Recomendaciones nutricionales de alimentos balanceados de perros y gatos registrados en Costa Rica
}

\author{
Mario Torres-Vargas $^{(i D}$ \& Rodolfo WingChig-Jones ${ }^{2}$ (D)
}

1. Universidad de Costa Rica, Escuela de Zootecnia, San José, Costa Rica; mtorres1388@gmail.com

2. Universidad de Costa Rica, Escuela de Zootecnia, Centro de Investigación en Nutrición Animal (CINA), San José, Costa Rica; rodolfo.wingching@ucr.ac.cr, rebeca.zamora@ucr.ac.cr

Received 18-XI-2020 • Corrected 19-V-2020 • Accepted 31-V-2021

DOI: https://doi.org/10.22458/urj.v13i2.3385

\begin{abstract}
Nutritional recommendations for dog and cat commercial food in Costa Rica". Introduction: The nutritional analysis of commercial food helps optimize its use and the pets' nutrition, and there are no recent analyses of this type for Costa Rica. Objective: To recommend pet foods according to the physiological state, level of activity and weight of cats and dogs in Costa Rica. Methods: Products registered at the government's Directorate for Animal Food were analyzed for nutritional information (2017) and animal requirements using three equations for cats and nine for dogs. Results: Of the average package recommendations, $71 \%$ would provide insufficient nutrition for dogs, and $63 \%$ insufficient nutrition for cats. Conclusions: Most commercial food products available in Costa Rica recommend amounts that are insufficient to meet the needs of many cats and dogs.
\end{abstract}

Keywords: Requirement, food consumption, body weight, physiological stages, commercial feed.
RESUMEN. Introducción: El análisis nutricional de alimentos comerciales ayuda a optimizar su uso y la nutrición de las mascotas, y no existen análisis recientes de este tipo para Costa Rica. Objetivo: Recomendar alimentos para mascotas de acuerdo al estado fisiológico, nivel de actividad y peso de perros y gatos en Costa Rica. Métodos: Los productos registrados en la Dirección de Alimentos para Animales del gobierno se analizaron para obtener información nutricional (2017) y los requisitos de los animales utilizando tres ecuaciones para gatos y nueve para perros. Resultados: De las recomendaciones de paquetes promedio, el $71 \%$ proporcionaría una nutrición insuficiente para los perros y el $63 \%$ una nutrición insuficiente para los gatos. Conclusiones: La mayoría de los productos alimenticios comerciales disponibles en Costa Rica recomiendan cantidades que son insuficientes para satisfacer las necesidades de muchos perros y gatos.

Palabras clave: Requerimiento, consumo de alimento, peso corporal, estadios fisiológicos, alimento comercial.

Los perros (Canis lupus familiaris) y gatos (Felis catus) durante el desarrollo de la humanidad fueron venerados, utilizados en la religión como figuras malignas, armas de guerra, cacería, protección, diversión, deporte y compañía entre otras (Gómez et al., 2007).

En Costa Rica, en el año 2013, se estimó una población de 1429544 perros y de 358566 gatos (Solís \& Ramírez, 2013). Lo que permite obtener para esa fecha una relación entre el número de habitantes y cada perro o gato de 30,30 y 7,60 (animales/100 habitantes), respectivamente (Población nacional 4717700 habitantes, SEPSA (2014)). En el caso de los perros con dueño, esta relación fue en el año 2015 de 27,01 perros por cada cien mil habitantes (WAP, 2016). La población de perros y otros animales domésticos con dueño, podría estar asociado a una solvencia económica 
en el núcleo familiar y a los beneficios terapéuticos, fisiológicos, psicológicos y sicosociales informados en la literatura al convivir con una mascota (Gómez et al., 2007).

La nutrición de las mascotas, representada por la acción de suministrar alimento, es uno de los factores limitantes para un desarrollo adecuado y una vida saludable. En Costa Rica, el 76\% de los hogares alimentan a sus perros con alimento balanceado comercial (WAP, 2016), una fuente directa a una alimentación balanceada; sin embargo, al haber variedad de opciones en el mercado, el comprador concentra la decisión en variables visuales y no nutricionales (Mesén, 2009); donde el cumplimiento de los requerimientos nutricionales del animal no es la prioridad. Situación que podría ocasionar problemas de salud, debido a que la nutrición de perros y gatos se ve afectada por factores específicos del animal (edad, estado fisiológico, actividad), de la dieta (desequilibrio de nutrimentos, deterioro, contaminación, adulteración) de las prácticas de alimentación que se implemente en el hogar (frecuencia de alimentación, método de alimentación) y variables ambientales (espacio, otros animales y calidad del entorno) (Balwin et al., 2010).

En Costa Rica, el etiquetado de los alimentos para animales está relacionado al Reglamento Técnico Centroamericano de Productos Utilizados en Alimentación Animal y Establecimientos (RTC 65.05.52:11, 2012) y la Ley para el Control de la Elaboración y Expendio de Alimentos para Animales (Ley 6883, 1983), donde solo se garantizan los valores de humedad (\%), proteína cruda (\%), extracto etéreo (\%), fibra cruda (\%), cenizas (\%), calcio (\%), fósforo (\%), sal (\%) y la energía digestible (Mcal/kg). Cada uno de los componentes de los cuales se garantiza su concentración (valores máximos y mínimos según corresponda), están asociado a la conservación del producto, reduciendo el crecimiento de microorganismos y a funciones nutricionales específicas (AAFCO, 2020). Es importante considerar, que la cantidad y digestibilidad de los nutrimentos que conforman estos alimentos, son los que definen el aporte nutricional y aprovechamiento de los diferentes alimentos balanceados comercializados en el país (Daumas et al., 2014).

En la descripción del aporte de nutrimentos de los alimentos comercializados en Costa Rica, se han realizado trabajos en la digestibilidad (pepsina) de la proteína cruda en alimentos para perro adulto y cachorro (Leiva et al., 2019); la caracterización de las grasas saturadas, monoinsaturadas y poliinsaturadas en alimentos para perro adulto, perro cachorro y gato adulto (Leiva et al., 2019), la descripción promedio de aminoácidos en alimentos extrusados para perro adulto y cachorro, y gatos (Leiva et al., 2019)y el perfil mineral de alimentos importados para perros y gatos (adulto y cachorro) (Cedeño, 2020) .

Una sustancia que no se describe en las etiquetas y presentan importancia en la nutrición de perros y gatos son las vitaminas. Es importante indicar que las materias primas utilizadas para la elaboración de los alimentos balanceados presentan un perfil de vitaminas específico para cada una, pero dependiendo del manejo y proceso que reciban de forma individual o durante la manufactura del alimento estas pueden inactivarse, debido a que son sensibles al calor, a la luz, humedad, minerales y a la oxidación (Trat et al., 2008).

Para calcular el requerimiento nutricional de los perros y gatos, se requiere el peso vivo expresado en kilogramos o libras según corresponda y el grado de actividad motora y física que presente el animal, con lo que, sumado al análisis de la condición corporal de los animales, permite hacer los ajustes necesarios para nutrir de forma adecuada a los animales. En el caso de la condición corporal de los perros y gatos, esta valoración fluctúa entre 5 y 9 puntos, según la casa de estudio (Brooks et al., 2014). En esta escala, se mide la acumulación de grasa en el cuerpo del animal, donde una calificación de 1, se asocia a faltante de grasa en la parte de las costillas, la base de la cola y la columna vertebral de los animales, y conforme se aumenta la valoración, los depósitos de grasa se hacen palpables y luego visibles (Brooks et al., 2014). Considerando valores entre uno a tres como animales con bajo peso, calificaciones de cuatro a cinco como peso saludable, de seis a siete como 
animales con sobrepeso y de ocho a nueve indican obesidad en el animal (van Herwijnen et al., 2020).

De igual manera, los nutricionistas animales cuando desarrollan un alimento balanceado, usan características promedio del animal (peso vivo, estado fisiológico y grado de actividad). Situación, que se contrapone, al análisis individual de las necesidades nutricionales de cada perro y gato, según las condiciones sociales, económicas y ambientales que les ofrece el propietario. Como dueños responsables, es importante conocer la cantidad precisa de calorías y nutrimentos que requieren las mascotas (requerimientos nutricionales), de esa manera sin importar el tipo, marca comercial o formulación del alimento balanceado, se ofrece a las mascotas la cantidad de alimento apropiada a su peso, actividad y estado fisiológico (Ward, 2015).

Por tal motivo, el objetivo de este trabajo fue conocer el perfil nutricional de los alimentos balanceados de perros y gatos que se encontraron inscritos desde agosto 2016 y hasta agosto 2017 en la Dirección de Alimentos para Animales (DAA/SENASA/MAG) y de generar una guía alimenticia que sirva de apoyo a las recomendaciones nutricionales descritas en los empaques de los alimentos balanceados comerciales según el estado fisiológico, grado de actividad y el peso de los animales.

\section{MATERIALES Y MÉTODOS}

Obtención de la Información: Generamos una base de datos de alimentos balanceados secos para perros y gatos registrados en la Dirección de Alimentos para Animales (DAA/SENASA/MAG) desde agosto del 2016 hasta agosto del 2017 (http://feednet.senasa.go.cr/consulta/index.htm). Entidad oficial, responsable del cumplimiento de las normas mínimas para comercialización de insumos alimenticios para animales de interés zootécnico en Costa Rica según Ley $\mathrm{N}^{\circ}$ 6883. Control Elaboración y Expendio Alimentos para Animales. Durante este periodo, la base de datos agrupó 955 alimentos para perros y 385 para gatos en diferentes etapas productivas.

Manipulación de la información: Organizamos la información según el estado fisiológico (cachorros, adultos, senior, prescripción, hembras gestantes y lactantes), peso del animal, la recomendación alimenticia según el peso y el análisis de garantía de los componentes nutricionales de cada alimento balanceado. Esta información se utilizó para calcular los gramos de alimento que se recomienda suministrar al animal (g/día) en la etiqueta, según su peso vivo. Por otro lado, con la misma información de peso $(\mathrm{kg})$ descrita en la etiqueta del alimento balanceado evaluado, se procedió a calcular el requerimiento energético (energía metabolizable) y con la información del análisis de garantía, se estimó la cantidad de alimento balanceado (g/día) que debería consumir el animal, para cubrir este requerimiento. Para el cálculo de la energía metabolizable, se utilizaron nueve ecuaciones para la estimación en perros y tres para gatos, las cuales permitieron determinar la cantidad de calorías que requiere el animal para mantenerse cada día (Tabla 1), según el grado de actividad. Posterior, al cálculo de las cantidades de alimento balanceado que la mascota debería consumir por día según las recomendaciones comerciales (QRC) y las calculadas para cumplir el requerimiento de energía ( $Q R E)$, se procedió a comparar estas cantidades de la siguiente manera: QRC-QRE, con el objetivo de determinar excesos o deficiencias en el consumo de alimento.

Cálculo de los carbohidratos (\%): Utilizamos la información nutricional disponible en el registro de cada alimento para perros y gatos que componen la base de datos, se procedió a calcular de forma individual los carbohidratos presentes en cada alimento registrado. Asumiendo que la sumatoria del contenido de agua $\left(\mathrm{H}_{2} \mathrm{O} \%\right)$, proteína cruda (PC \%), extracto etéreo (EE \%) y/o grasa, fibra cruda (FC \%), cenizas (Ce \%) y carbohidratos (C\%) equivale al $100 \%$, el contenido estimado de 
los carbohidratos presentes se calculó por diferencia, donde al $100 \%$ se le resto el valor resultante de la sumatoria del contenido de agua, proteína cruda, extracto etéreo, fibra cruda y ceniza ( $C$ $\%=100-(\mathrm{H}+\mathrm{PC}+\mathrm{FC}+\mathrm{EE}+\mathrm{Ce}))$.

Análisis de la información: El valor resultante de la operación (QRC-QRE) se interpretó de la siguiente manera; si es un valor positivo, se entiende que la recomendación comercial establece una sobredosificación de alimento, según lo obtenido por su requerimiento, en caso contrario un valor negativo, subestima el requerimiento energético del animal, lo que podría provocar desnutrición o desbalances nutricionales en los animales, por lo cual, habría que variar la cantidad de alimento suministrado según sea el caso. Cuando el resultado de la operación es cero, la recomendación comercial satisface el requerimiento nutricional según el peso vivo. Por tal motivo, se generó una guía que permitirá considerar un rango de corrección sobre la cantidad de alimento promedio que se recomienda suministrar, que contempla el peso y el estado fisiológico del animal (perro o gato), según la ecuación de predicción utilizada para estimar el requerimiento de la energía metabolizable de la mascota.

TABLA 1

Criterios utilizados para el cálculo de ingesta de energía metabolizable por día para perros y gatos según su peso vivo expresado en kilogramos o libras y el grado de actividad

\begin{tabular}{|c|c|c|}
\hline Especie & Fórmula Utilizada* & Fuente \\
\hline \multirow{6}{*}{ Canis lupus familiaris } & $E M /$ día $=132 \times\left(P_{\mathrm{kg}}\right)^{0,75}$ & NRC (1974) \\
\hline & $\mathrm{EM}=\mathrm{K} \times \mathrm{P}_{\mathrm{kg}}{ }^{0,67 * *}$ & NRC (1985) \\
\hline & $E M=P_{\mid b} \times\left(33-1 / 4 P_{l b}\right)$ & \multirow{2}{*}{ Jurgens (1997) } \\
\hline & $\mathrm{EM}=18 \times \mathrm{P}_{\mathrm{lb}}$ & \\
\hline & $\mathrm{EM}=70 \mathrm{kcal} \times\left(\mathrm{P}_{\mathrm{kg}}\right)^{0,75^{* * *}}$ & \multirow{2}{*}{ NRC (2006) } \\
\hline & $\mathrm{EM}=144 \mathrm{Kcal} \times\left(P_{\mathrm{kg}}\right)^{0,75^{* * * *}}$ & \\
\hline \multirow{3}{*}{ Felix catus } & $E M=60 \mathrm{kcal} \times\left(P_{\mathrm{kg}}\right)^{\mathrm{a}}$ & \multirow{3}{*}{ NRC (2006) } \\
\hline & $\mathrm{EM}=70 \mathrm{kcal} \times\left(\mathrm{P}_{\mathrm{kg}}\right)^{\mathrm{e}}$ & \\
\hline & $\mathrm{EM}=80 \mathrm{kcal} \times\left(\mathrm{P}_{\mathrm{kg}}\right)^{\circ}$ & \\
\hline
\end{tabular}

*P= peso corporal del animal en libras (lb) o kilogramos (kg) según corresponda.

$* * \mathrm{~K}=$ constante de actividad: 132 : inactivo, 145: activo, 200: muy activo, 300: alto rendimiento

$* * *=$ poco espacio; $* * * *$ Mayor Espacio y Actividad

${ }^{a}=$ Sedentario, ${ }^{e}=$ moderadamente activo; ${ }^{\circ}=$ activo 


\section{RESULTADOS}

Los alimentos para gatos y perros analizados presentan para cada variable nutricional, valores que se traslapan según el uso recomendado por el fabricante (Tabla 2, Tabla 3). De igual manera, se observa la variación en una misma variable la concentración de la misma, la cual afecta el aporte nutricional de los alimentos con registro inscrito durante el periodo de evaluación de esta investigación.

Al valorar la fracción inorgánica de los alimentos registrados, para gatos y perros, se determinan relaciones entre calcio y fósforo $(\mathrm{Ca} / \mathrm{P})$ en alimentos para gatos de 1:1 hasta 2:1. Mientras que, en los alimentos para perro, esta relación fluctuó entre 1,17 a 1,45.

Al evaluar los aportes proporcionales de energía de las fracciones de proteína, grasa (EE) y carbohidratos, no se determinó una tendencia en los alimentos para gatos, donde según el grupo meta, el $57,14 \%$ de estos presenta un aporte mayoritario por los compuestos agrupados en el extracto etéreo, mientras que, solo en adultos y animales Senior, el mayor aporte lo dan las proteína y carbohidratos respectivamente (Tabla 2). Caso contrario sucede en los alimentos para perros (Tabla 3), donde en todos los casos evaluados, la principal fuente de energía, son los carbohidratos presentes en los alimentos evaluados.

Al contrastar las cantidades de alimento que se debe ofrecer a los animales por día, se encuentran diferencias entre lo recomendado y lo requerido según el cálculo de los requerimientos de energía del animal según su peso, y posterior transformación a gramos de alimento a consumir por día. Para la evaluación de los alimentos para gatos (Tabla 4), se determina que el 62,96\% (17/27 dosificaciones de alimento promedio) suministran una cantidad menor a la requerida por el perro, mientras que, la evaluación de las recomendaciones de los alimentos para perro fue de $71,43 \%$ de las recomendaciones promedio (45/63 dosificaciones de alimento) (Tabla 5).

Las ecuaciones utilizadas, permiten demostrar la importancia de considerar la actividad de los gatos y perros, en el cálculo del requerimiento diario de la energía metabolizable para un adecuado cuidado del animal de compañía. La diferencia en los resultados está asociada al perfil nutricional de los alimentos evaluados según el uso recomendado del fabricante y a la consideración de la actividad en el cálculo del requerimiento. 


\section{TABLA 2}

Concentraciones promedio de nutrimentos de alimentos para gatos registrados en la base de datos de la Dirección de Alimentos para Animales desde agosto del 2016 hasta agosto del 2017 según el tipo de animal.

\begin{tabular}{|c|c|c|c|c|c|c|c|c|}
\hline Nutrimentos & & Cachorros & Adultos & Senior & Prescripción & Por Raza & $\begin{array}{l}\text { Cachorro, lactantes } \\
\text { gestantes, adultos }\end{array}$ & $\begin{array}{l}\text { Adulto y } \\
\text { cachorro }\end{array}$ \\
\hline $\mathbf{n}$ & & 26 & 166 & 18 & 60 & 18 & 23 & 74 \\
\hline \multirow{2}{*}{ Humedad (\%) } & Promedio & 10,83 & 10,78 & 10,46 & 9,51 & 7,47 & 9,10 & 10,22 \\
\hline & Rango & $5,2-13,0$ & $6,0-13,0$ & $7,0-13,0$ & $7,0-13,0$ & $7,0-8,5$ & $7,0-10,0$ & $10,0-12,0$ \\
\hline \multirow{2}{*}{ Proteína (\%) } & Promedio & 34,12 & 34,88 & 32,27 & 29,33 & 28,80 & 33,84 & 34,37 \\
\hline & Rango & $21,0-42,0$ & $23,0-45,0$ & $23,0-42,0$ & $21,0-45,0$ & $28,0-30,0$ & $30,0-41,0$ & $26,0-44,0$ \\
\hline \multirow{2}{*}{ Extracto Etéreo (\%) } & Promedio & 15,0 & 12,76 & 12,50 & 15,66 & 20 & 18,93 & 15,41 \\
\hline & Rango & $7,0-21,0$ & $6,0-26,0$ & $6,0-24,0$ & $7,0-23,0$ & - & $9,0-24,0$ & $8,0-20,0$ \\
\hline \multirow{2}{*}{ Fibra Cruda (\%) } & Promedio & 4,22 & 5,91 & 4,89 & 5,13 & 5,39 & 3,12 & 4,01 \\
\hline & Rango & $2,0-7,7$ & $2,0-16,5$ & $2,0-10,0$ & $6,5-16,0$ & $3,7-7,0$ & $2,0-4,0$ & $3,0-6,0$ \\
\hline \multirow{2}{*}{ Carbohidratos (\%) } & Promedio & 27,47 & 27,82 & 39,89 & 33,49 & 38,33 & 35,00 & 28,15 \\
\hline & Rango & $6,3-58,3$ & $0,0-57,0$ & $11,0-62,0$ & $0,0-57,0$ & $34,5-41,3$ & $21,0-52,0$ & $5,0-52,4$ \\
\hline \multirow{2}{*}{ Cenizas (\%) } & Promedio & 8,36 & 7,86 & 7,63 & 6,89 & - & - & 7,85 \\
\hline & Rango & $6,5-10,0$ & $6,0-10,0$ & $7,0-8,5$ & $6,50-7,30$ & - & - & $6,0-13,0$ \\
\hline \multirow{2}{*}{ Calcio (\%) } & Promedio & 0,94 & 0,89 & 1,02 & 0,74 & 0,74 & 1,04 & 1,04 \\
\hline & Rango & $0,5-1,6$ & $0,20-2,50$ & $0,4-2,5$ & $0,5-1,0$ & $0,6-0,9$ & $0,8-1,8$ & $0,8-1,8$ \\
\hline \multirow{2}{*}{ Fósforo (\%) } & Promedio & 0,79 & 0,72 & 0,79 & 0,76 & 0,66 & 0,84 & 0,80 \\
\hline & Rango & $0,5-1,0$ & $0,1-1,2$ & $0,4-1,1$ & $0,2-1,3$ & $0,5-0,8$ & $0,5-1,2$ & $0,5-1,0$ \\
\hline \multirow{2}{*}{ Sal (\%) } & Promedio & 0,42 & 0,59 & 0,48 & 1,21 & 0,65 & 0,90 & 0,41 \\
\hline & Rango & $0,0-1,1$ & $0,0-2,4$ & $0,1-1,1$ & $0,2-2,16$ & $1,0-1,2$ & $0,2-0,9$ & $0,2-0,91$ \\
\hline \multirow{2}{*}{ EM (kcal) } & Promedio & 3672 & 3613 & 3636 & 3772 & 4203 & 3693 & 3693 \\
\hline & Rango & $3028-4449$ & $2860-4588$ & $2866-4089$ & $3176-4292$ & $4143-4272$ & $3250-4170$ & $3250-4170$ \\
\hline \multirow{3}{*}{$\begin{array}{l}\text { Aporte proporcional* } \\
\text { de la energía }\end{array}$} & E-PC (\%) & 34,81 & 37,23 & 31,48 & 29,08 & 24,89 & 29,47 & 34,40 \\
\hline & E- EE (\%) & 37,16 & 33,08 & 29,61 & 37,71 & 41,98 & 40,04 & 37,45 \\
\hline & E-Cho's\%) & 28,03 & 29,69 & 38,91 & 33,21 & 33,13 & 30,48 & 28,15 \\
\hline
\end{tabular}

*Fraccionamiento de la energía según su origen. Proveniente de la proteína (E-PC\%), del extracto etéreo (E-EE\%) y de los carbohidratos (E-Cho's\%) 


\section{TABLA 3}

Concentraciones promedio de nutrimentos de alimentos para perros registrados en la base de datos de la Dirección de Alimentos para Animales desde agosto del 2016 hasta agosto del 2017 según el tipo de animal.

\begin{tabular}{|c|c|c|c|c|c|c|c|}
\hline Nutrimentos & & Cachorros & Adultos & Prescripción & Senior & $\begin{array}{c}\text { Cachorro y hembras } \\
\text { lactantes }\end{array}$ & $\begin{array}{l}\text { Adulto y } \\
\text { cachorro }\end{array}$ \\
\hline $\mathbf{n}$ & & 199 & 492 & 145 & 55 & 19 & 45 \\
\hline \multirow{2}{*}{ Humedad (\%) } & Promedio & 11,01 & 10,95 & 10,34 & 10,85 & 10,92 & 10,20 \\
\hline & Rango & $10,3-12,0$ & $10,2-11,7$ & $7,0-12,0$ & $10,0-12,0$ & $9,0-12,0$ & $10,0-11,0$ \\
\hline \multirow{2}{*}{ Proteína (\%) } & Promedio & 27,2 & 23,10 & 24,61 & 24,79 & 26,94 & 29,18 \\
\hline & Rango & $25,4-29,1$ & $19,7-25,3$ & $19,0-40,0$ & $22,2-26-7$ & $25,7-28,0$ & $24,0-34,0$ \\
\hline \multirow{2}{*}{ Extracto Etéreo (\%) } & Promedio & 13,6 & 11,43 & 13,06 & 11,12 & 14,61 & 14,75 \\
\hline & Rango & $10,0-16,0$ & $8,3-14,1$ & $8,0-22,0$ & $7,0-13,0$ & $7,0-20,0$ & $12,0-16,7$ \\
\hline \multirow{2}{*}{ Fibra Cruda (\%) } & Promedio & 3,73 & 4,44 & 6,51 & 4,83 & 3,49 & 5,49 \\
\hline & Rango & $3,3-4,6$ & $3,9-5,0$ & $3,5-16,0$ & $2,9-7,2$ & $2,3-5,0$ & $3,0-12,0$ \\
\hline \multirow{2}{*}{ Carbohidratos (\%) } & Promedio & 38,39 & 43,98 & 40,04 & 42,28 & 37,51 & 34,98 \\
\hline & Rango & $35,4-44,0$ & $39,9-49,5$ & $22,0-50,5$ & $38,1-46,4$ & $33,7-40,0$ & $27,2-41,0$ \\
\hline \multirow{2}{*}{ Cenizas (\%) } & Promedio & 6,13 & 6,09 & 5,44 & 6,12 & 6,69 & 5,4 \\
\hline & Rango & $5,0-9,0$ & $5,0-8,4$ & $5,0-7,5$ & $5,0-9,0$ & $5,0-10,0$ & $5,0-7,0$ \\
\hline \multirow{2}{*}{ Calcio (\%) } & Promedio & 1,16 & 1,09 & 0,90 & 0,89 & 1,03 & 0,99 \\
\hline & Rango & $0,9-1,5$ & $0,7-1,6$ & $0,7-1,1$ & $0,7-1,0$ & $0,9-1,1$ & $0,7-1,2$ \\
\hline \multirow{2}{*}{ Fósforo (\%) } & Promedio & 0,80 & 0,76 & 0,65 & 0,66 & 0,88 & 0,75 \\
\hline & Rango & $0,6-1,1$ & $0,5-1,0$ & $0,5-0,9$ & $0,4-0,8$ & $0,7-1,0$ & $0,4-0,9$ \\
\hline \multirow{2}{*}{ Sal (\%) } & Promedio & 0,52 & 0,48 & 0,59 & 0,41 & 0,76 & 0,49 \\
\hline & Rango & $0,2-0,7$ & $0,2-0,8$ & $0,1-0,8$ & $0,2-0,5$ & $0,5-1,0$ & $0,2-0,8$ \\
\hline \multirow{2}{*}{ EM (kcal) } & Promedio & 3445 & 3319 & 3373 & 3293 & 3492 & 3499 \\
\hline & Rango & $3142-3603$ & $3059-3478$ & $2657-4040$ & $3045-3495$ & $2905-3908$ & $3120-3670$ \\
\hline \multirow{3}{*}{$\begin{array}{l}\text { Aporte proporcional* de } \\
\text { la energía }\end{array}$} & E-PC (\%) & 27,58 & 24,36 & 25,54 & 26,35 & 26,96 & 27,80 \\
\hline & E- EE (\%) & 33,49 & 29,27 & 32,91 & 28,71 & 35,51 & 34,12 \\
\hline & E-Cho's(\%) & 38,93 & 46,37 & 41,55 & 44,94 & 37,54 & 38,08 \\
\hline
\end{tabular}

*Fraccionamiento de la energía según su origen. Proveniente de la proteína (E-PC\%), del extracto etéreo (E-EE\%) y de los carbohidratos (E-Cho's\%) 
TABLA 4

Promedio de las diferencias entre las recomendaciones alimenticias y requerimientos de alimentos para gatos registrados en la base de datos de la Dirección de Alimentos para Animales entre agosto del 2017 y agosto del 2018 según el tipo de animal

\begin{tabular}{|c|c|c|c|c|}
\hline \multirow[t]{2}{*}{ Tipo de animal } & \multirow[t]{2}{*}{$\mathbf{n}$} & Sedentarios & $\begin{array}{c}\text { Moderadamente } \\
\text { activo }\end{array}$ & Activos \\
\hline & & \multicolumn{3}{|c|}{ (g/día) } \\
\hline Cachorros & 26 & +32 & +25 & +17 \\
\hline Adultos & 166 & -4 & -17 & -30 \\
\hline Senior & 18 & -15 & -31 & -47 \\
\hline Por Raza (Persa) & 18 & -5 & -14 & -63 \\
\hline Cachorro, gestantes y lactantes: Cachorro & 15 & +39 & +36 & +33 \\
\hline Cachorro, gestantes y lactantes: Adulto & 26 & +58 & +47 & +36 \\
\hline Cachorro, gestantes y lactantes: Gestante & 21 & +89 & +74 & +60 \\
\hline Cachorro, gestantes y lactantes: Lactantes & 21 & +159 & +111 & +64 \\
\hline Adulto y cachorro & 74 & +14 & $+0,5$ & -13 \\
\hline
\end{tabular}

TABLA 5

Promedio de las diferencias entre las recomendaciones alimenticias y requerimientos de alimentos para perros registrados en la base de datos de la Dirección de Alimentos para Animales desde agosto del 2017 hasta agosto y agosto del 2018 según el tipo de animal.

\begin{tabular}{|c|c|c|c|c|c|c|c|}
\hline \multirow[t]{3}{*}{ Fórmula Utilizada } & Adultos & Cachorros & $\begin{array}{l}\text { Adulto/ } \\
\text { Cachorro }\end{array}$ & $\begin{array}{c}\text { Adulto/Cachorro } \\
\text { /Senior }\end{array}$ & $\begin{array}{c}\text { Cachorro y } \\
\text { hembra } \\
\text { Lactante }\end{array}$ & Senior & Prescripción \\
\hline & \multicolumn{7}{|c|}{ (g/día) } \\
\hline & 492 & 199 & 40 & 5 & 19 & 55 & 145 \\
\hline$E M=132 \times(P)^{0,75 *}$ & -106 & +4 & -54 & -227 & -54 & -135 & -154 \\
\hline$E M=X(33-1 / 4 X) * *$ & +7 & +89 & +48 & -25 & +39 & -20 & -18 \\
\hline$E M=18 X^{* * *}$ & -49 & +21 & -51 & -195 & -47 & -125 & -118 \\
\hline$E M=132 \times P_{k g}^{0,67}$ & -12 & +76 & +29 & -80 & +10 & -53 & -44 \\
\hline 70 kcal EM x $\left(P_{k g}\right)^{0,75}$ & +83 & +154 & +110 & +48 & +88 & +36 & +59 \\
\hline 144 kcal EM x $\left(P_{k g}\right)^{0,75}$ & -140 & -25 & -85 & -280 & -81 & -168 & -195 \\
\hline$E M=145 \times P_{k g}^{0,67}$ & -42 & +50 & +3 & -123 & -14 & -81 & -78 \\
\hline$E M=200 \times P_{k g} 0,67$ & -170 & -54 & -108 & -304 & -113 & -199 & -222 \\
\hline$E M=300 \times P_{k g}^{0,67}$ & -400 & +244 & -346 & -638 & -293 & -413 & -483 \\
\hline
\end{tabular}

-Las cantidades positivas y negativas indican la cantidad a ofrecer de alimento ya sea mayor o menor a la cantidad indicada en la etiqueta del alimento balanceado. ${ }^{*} \mathrm{P}=$ peso corporal del animal; **animales con un peso vivo entre 5 y $65 \mathrm{lb} ; * *$ *animales con peso vivo superior a las $65 \mathrm{lb}$ 


\section{DISCUSIÓN}

Las recomendaciones nutricionales de los alimentos balanceados secos presentes en el mercado nacional y que se encontraron registrados durante los meses de evaluación, presentan diferencias entre lo recomendado y lo que deben consumir de forma diaria, diferencia que se asocia de manera integral a la forma de estimar los requerimientos energéticos, el estado fisiológico de los animales, la condición corporal, el medio ambiente en el que se moviliza, la socialización con otros animales y el tipo de materia prima utilizada (información no evaluada en este trabajo) (TordaOrsolya et al., 2020).

Si esta práctica es constante a través del tiempo, se van a evidenciar procesos de desnutrición o sobre peso y/o obesidad (Yam et al., 2016). Situación, que afecta la salud del animal, y que podría hacer pensar a los dueños de los animales, que el alimento no es el adecuado, cuando es más un error de suministro de alimento (aporte de nutrimentos).

Una práctica como dueño responsable de un perro o gato, es el pesaje de los animales una vez al mes, lo que va permitir generar una curva de crecimiento (edad contra peso), y comparar este comportamiento contra la curva ideal de la raza o del patrón racial predominante (Salt et al., 2020; Cave et al., 2018). Lo que va permitir generar ajustes en la cantidad de alimento ofrecida ( $\pm 5 \% /$ día) según el comportamiento del peso y valorar el aumento o disminución de la actividad física, por parte del profesional en nutrición animal.

Al contrastar las concentraciones promedio en los alimentos evaluados contra los niveles recomendados de estos en alimentos para perros y gatos, se determinan que todos se encuentran dentro del rango de aceptabilidad para cada especie (Hand, 2000). Es importante considerar, que los animales de interés zootécnico, no consumen porcentajes, por lo que, dependiendo de las características del alimento se hacen los ajustes en el consumo, con el fin de garantizar los gramos, miligramos o kilocalorías, requeridas por día.

En el caso de la relación humedad/materia seca, esta se vincula a un efecto de concentración o dilución de los componentes presentes y que el crecimiento de microrganismos se reduce con valores menores a $13 \%$ de humedad (Olatunde \& Atungulu, 2018). Situación que se complementa con el tipo de empaque, el cual debe de aislar el alimento de la humedad del ambiente y protegerlo de su entorno, este valor de $13 \%$ de humedad, se asocia al microambiente que se debe generar y mantener a lo interno del empaque cerrado y después de abierto, para mantener su calidad microbiológica (Wrap, 2009).

Para el caso de la proteína y el extracto etéreo (o grasa) los niveles recomendados fluctúan entre 35 a $50 \%$ y 18 a 35\% para gatos y 15 a $30 \%$ y 10 a $20 \%$ en perros respectivamente (Hand et al., 2000). Estos nutrimentos tienen una connotación importante, debido a los aportes de aminoácidos (arginina, glutamina, isoleucina, leucina, lisina, metionina, fenilalanina, taurina, treonina, triptófano y valina) y ácidos grasos (ácido eicosapentaenoico, ácido docosahexaenoico, ácido alfa-linolénico, ácido araquidónico y ácido linoleico) respectivamente, que el perro o el gato no pueden sintetizar a partir de otras moléculas (nutrimento esencial) (Hand et al., 2000), por lo cual se debe garantizar el consumo de forma diaria. En el caso de los ácidos grasos, estos se asocian a la acción antiinflamatoria, oxigenación de células, esfuerzo físico, integridad de la piel, pelaje y reproducción (NRC, 2006). Mientras que los aminoácidos a la construcción de las fibras musculares, la sangre, piel, hormonas y enzimas (NRC, 2006).

En el alimento balanceado para gatos, es importante que se garantice la concentración de taurina (1 000 ppm/al día), debido a que no lo puede sintetizar a partir de otras moléculas, y participa en el desarrollo del organismo, volumen celular y en la vista de los animales (Ripps \& Shen, 2012). Por tal motivo, se debe prestar atención en los hogares, cuando se tienen perros y gatos, a que el gato no consuma el alimento para perros, ya que, en estas formulaciones, no se presta 
atención a la taurina, debido a que el perro lo puede sintetizar a partir de cistina.

La fibra según su solubilidad presenta funciones físicas para el adecuado funcionamiento del tracto digestivo (baja solubilidad) como la celulosa y la lignina, como prebióticos, los cuales estimulan el crecimiento de bacterias benéficas (pectina, betaglucanos y fructooligosacáridos) (Ephraim \& Jewell, 2020) y los almidones y azúcares, que presentan una alta solubilidad y aporte energético al animal (NRC, 2006). En este trabajo, las etiquetas presentan un valor total de fibra cruda cercano al ideal para ambas especies, el cual es menor o igual a cinco.

El contenido de carbohidratos no es obligado garantizarlo, debido a que no es esencial para perros y gatos (Corsato-Alvarenga \& Aldrich, 2020), se genera en este trabajo una valoración del contenido de esta fracción en el alimento balanceado según la especie y el estado fisiológico. Nutrimento que se obtiene al restar al $100 \%$, el contenido de humedad, proteína cruda, fibra, extracto etéreo y cenizas, fracción que se afecta de forma directa con el contenido de cada variable nutricional.

Otro aspecto importante a recordar, es la diferencia en la fisiología de los perros y gatos, donde el primero es omnívoro, y el segundo carnívoro estricto (Di Cerbo et al., 2017) por lo que el origen de la materia prima que aporta los carbohidratos en el alimento, va estar en función de los ingredientes utilizados en la formulación y su calidad.

Es importante considerar, que en el proceso de domesticación del gato, este presenta una mejora en el aprovechamiento de los carbohidratos debido a cambios metabólicos y anatómicos en el tracto gastrointestinal (Curso-Almeida, 2020), como el uso de aminoácidos como fuente de energía por gluconeogénesis (Di Cerbo et al., 2017). En cambio, en el perro se desarrollan los genes AMY2B, MGAM y SGLT1, que le permiten la digestión de los almidones y el uso de la glucosa, la síntesis de niacina, taurina y arginina en el sistema digestivo (Di Cerbo et al., 2017).

Los minerales son importantes en el desarrollo de los perros y gatos por sus funciones como componentes estructurales de órganos y tejidos, mantenimiento de la presión osmótica en líquidos, balance ácido base, contracciones musculares, cofactores y catalizadores de sistemas enzimáticos y hormonales (Hand et al., 2000; NRC, 2006).

En el caso de las etiquetas evaluadas, la fracción de cenizas, calcio (Ca), fósforo (P) y sal $(\mathrm{NaCl})$, hace referencia a esta fracción. Otros minerales que presentan importancia en la nutrición de perros y gatos son el potasio, magnesio, hierro, zinc, manganeso, yodo, boro, cromo y selenio. En ambas especies se recomiendan relaciones de $\mathrm{Ca} / \mathrm{P}$ de 1:1 a 1:5 en gatos y de 1:1 a 2:1 en perros (Hand et al., 2000), relaciones importantes en el desarrollo óseo de los animales y para evitar antagonismo con otros minerales.

La energía metabolizable es el resultado de la sumatoria de los aportes de las proteínas, al utilizar la parte de carbono de los aminoácidos en el metabolismo (Maynard et al., 1981), los carbohidratos y las grasas, con valores de 8,5, 3,5 y 3,5 kcal de EM/g respectivamente.

En estudios realizados donde se mide el consumo voluntario de perros y gatos, informan de la proporción de la energía según el origen de esta, que permite optimizar el consumo de materia seca de forma voluntaria, en el caso de perros esta relación de proteína:grasa:carbohidratos es de 38:59:3 y en gatos es de 52:36:12 (Roberts et al., 2018), valores diferentes a los calculados para los alimentos para perros $(26,43: 32,33: 41: 24)$ y gatos $(31,62: 36,72: 31,66)$ con registro activo en el país durante la evaluación, donde los alimentos evaluados, en promedio recargan el aporte energético en los alimentos en las fuentes de origen vegetal.

Las recomendaciones alimenticias en productos alimenticos comerciales presentan su cálculo en un animal promedio, modelo de predicción que no considera la actividad física del animal, si se encuentra esterilizado (Oberbauer et al., 2019), si es una hembra o un macho (Bjørnvad et al., 2019), si convive con otros animales, el espacio físico en el cual se mantiene (Torda-Orsolya et al., 2020), si reciben aperitivos o meriendas (Pearl et al., 2020) y las características de los dueños (van 
Herwijnen et al., 2020).

De igual manera, la costumbre del ser humano a ofrecer una cantidad adicional de alimento, por encima de lo recomendado, estimula al animal a aumentar el consumo voluntario (Kersbergen et al., 2019), lo que podría promover una situación de suministro excesivo de nutrimentos, que provoca en los animales de compañía el sobrepeso, diabetes, problemas musculares (van Herwijnen et al., 2020), problemas cardiorespiratorios y desordenes urinarios (Roberts, 2017).

La información descrita en este estudio evidencia la diferencia entre recomendación y requerimiento (Zicker, 2008). La misma, es de utilidad para ayudar a las personas dueñas de perros y gatos a ofrecer las cantidades adecuadas de alimento de forma diaria, por medio de la evaluación de las recomendaciones comerciales y el cálculo de los requerimientos, sin importar el tipo de alimento o el estado fisiológico del animal, de una manera más exacta, ajustando las raciones de acuerdo a la información descrita y combinado a prácticas de ejercicio y juego para la mascota (perros y gatos), con la intención de disminuir, afectaciones a la salud; y tener una mascota sana y bien nutrida (Heuberger \& Wakshlag, 2011). Es importante recordar, que lo discutido en este trabajo y las recomendaciones planteadas, no sustituyen, el análisis individual de cada animal de compañía, por un profesional en nutrición animal.

\section{AGRADECIMIENTOS}

Agradecemos a la Vicerrectoría de Investigación de la Universidad de Costa Rica el apoyo brindado al proyecto de Investigación739-B762 Comparación de los requerimientos de perros y gatos con las recomendaciones de alimentación en las etiquetas de alimentos comerciales, desarrollado en el Centro de Investigación en Nutrición Animal (CINA).

\section{ÉTICA, CONFLICTO DE INTERESES Y DECLARACIÓN DE FINANCIAMIENTO}

Los autores, Mario Torres Vargas y Rodolfo WingChing-Jones declaramos haber cumplido con todos los requisitos éticos y legales pertinentes, tanto durante el estudio como en el manuscrito; que no hay conflictos de interés de ningún tipo, y que todas las fuentes financieras se detallan plena y claramente en la sección de agradecimientos. Asimismo, están de acuerdo con la versión editada final del documento. El respectivo documento legal firmado se encuentra en los archivos de la revista.

La declaración de la contribución de cada autor al manuscrito fue la siguiente: M.T.V. recolección, análisis y escritura de la información. Mientras que R.W.CH.J, participo en el análisis, escritura del artículo. 


\section{REFERENCIAS}

AAFCO (2020). Animal feed labeling guide. Association of Amercian Feed Control Officials (AAFCO). Headquarters Office 1800 S. Oak Street, Suite 100 Champaign, IL 61820-6974, USA. https://bit.ly/3qFbhYI

Balwin, K., Bartges, J., Buffington, T., Freeman, L., Grabow, M., Legred, J., \& Ostwald, D. (2010). Guías para la Evaluación Nutricional de perros y gatos de la Asociación Americana Hospitalaria de Animales (AAHA). Journal of the American Animal Hospital Association, 46, 4. https://www.aaha.org/globalassets/02-guidelines/nutritionalssessment/nag_spanish_color.pdf

Bjørnvad, C.R., Gloor, S., Johansen, S.S., Sandøe, P., \& Lund, T.B. (2019). Neutering increases the risk of obesity in male dogs but not in bitches - Across-sectional study of dog- and owner-related risk factors for obesity in Danish companion dogs. Preventive Veterinary Medicine, 170, 104730. https://doi.org/10.1016/j.prevetmed.2019.104730

Brooks, D., Churchill, J., Fein, K., Linder, D., Michel, K., Tudor, K., Ward, E., \& Witzel, A. (2014). Weight management guide lines for dogs and cats. Journal Amercan Animal Hospital Association, 50, 1-11. https://doi.org/10.5326/JAAHAMS-6331

Cave, N. J.; Bridges, J. P.; Weidgraaf, K., \& Granville-Thomas, D. (2018). Non linear mixed models of growth curves from domestics hort hair cats in a breeding colony, housed in a seasonal facility to predict obesity. Journal of Animal Physiology and Animal Nutrition, 102, 1390-1400. https://doi.org/10.1111/jpn.12930

Cedeño, A. (2020). Perfil mineral de los alimentos para mascotas importados y verficación de su contenido nutricional, según su etiqueta de análisis garantizado. (Tesis, Licenciatura). Universidad de Costa Rica, Costa Rica. http://www.zootecnia.ucr.ac.cr/index.php/es/asuntos-estudiantiles/tesis-para-consulta

Corsto-Alvarenga, I., \& Aldrich C., (2020). Starch characterization of comercial extruded dry petfoods. Translational Animal Science,4,1017-1022. https://doi.org/ 10.1093/tas/txaa018

Curso-Almeida, P. (2020). Effects of pea starch yeast fermentation on glycemic index, palatability, metabolic status and intestinal health of dogs and cats fed a pea based diet. (Thesis, Master science) University of Sakatchewan, Canada. $\quad$ https://harvest.usask.ca/bitstream/handle/10388/12697/CURSOALMEIDA-THESIS2020.pdf?sequence=1\&isAllowed=y

Daumas, C., Paragon B.M., Thorin, C., Martin, L., Dumon, H., Ninet, S. \& Nguyen, P. (2014). Evaluation of eight commercial dog diets. Journal of Nutritional Science, 3(e63), 1-5. https://doi.org/10.1017/jns.2014.65

Di Cerbo, A., Morales-Medina J. C., Palmieri, B., Pezzutto, F., Cocco, R., Flores, G., \& lannitti, T. (2017). Functional foods in pet nutrition: Focus on dogs and cats. Research in Veterinary Science, 112, 161-166. http://dx.doi.org/10.1016/j.rvsc.2017.03.020

Ephraim, E., \& Jewell D. (2020). Effect of added dietary betaine and soluble fiber on metabolites and fecal microbiome in dog with early renal disease. Metabolites, 10, 0370; https://doi.org/10.3390/metabo10090370

Gómez L., Atehortua, C., \& Orozco S. (2007). La influencia de las mascotas en la vida humana. Revista Colombiana de Ciencias Pecuarias, 20, 377-386.

Hand, M., Thatcher, C., Remillard, R., \& Roudebush, P. (2000). Nutrición clínica en pequeños animales (Small Animal Clinical Nutrition). Buenos Aires: Editorial Inter-Médica

Heuberger, R. \& Wakshlag, J. (2011). The relationship of feeding patterns and obesity in dogs. Journal of Animal Physiology and Animal Nutrition, 95, 98-105. https://doi.org/ 10.1111/j.1439-0396.2010.01024.x

Jurgens, M. (1997). Animal Feeding and Nutrition. $8^{\circ}$ Edición. lowa: Kendall/Hunt Publishing Company. 
Kersbergen, I., German, A., Westgarth, C., \& Robinson, E. (2019). Portion size and meal consumption in domesticated dogs: An experimental study. Physiology \& Behavior, 204, 174-179. https://doi.org/10.1016/j.physbeh.2019.02.034

Leiva, A., Molina, A., Redondo-Solano, M., Artavia, G., Rojas-Bogante, L., \& Granados-Chinchilla, F. (2019). Pet food quality assurance and safety and quality assurance survey with in the Costa Rican petfood industry. Animals, 9, 980; https://doi.org/10.3390/ani9110980

Ley $\mathrm{N}^{\circ}$ 6883. (1983). Ley $N^{\circ}$ 6883. Para el Control de la Elaboración y Expendio de Alimentos para Animales. http://www.pgrweb.go.cr/SCIJ/Busqueda/Normativa/Normas/nrm_texto_completo.aspx?param1=NRTC\&nVal or1=1\&nValor $2=17703 \&$ nValor3 $=18890 \&$ strTipM $=\mathrm{TC}$

Maynard, L., Loosli. J., Hintz, H., \& Warner, R. (1981). Nutrición Animal. Sétima edición. USA: McGraw Hill Book Co.

Mesén, M. (2009). Desarrollo de la categoría de mascotas en supermercados versus tiendas de mascotas en Costa Rica. (Trabajo de licenciatura). Universidad Latinoamericana de Ciencia y Tecnología. San José, Costa Rica.

National Research Council (NRC). (1974). Nutrient Requirements of Dogs. Washinton, D.C., USA: National Academy Press.

National Research Council (NRC). (1985). Nutrient Requirements of Dogs. Washinton, D.C., USA: National Academy Press.

National Research Council (NRC). (2006). Nutrient Requirements of Dogs and Cats. Washinton, D.C., USA: National Academy Press.

Oberbauer, A., Belanger, J., \& Famula, T. (2019). A review of the impact of neuter status on expresión of in herited conditions in dogs. Reviews. Frontiers in Veterinary Science, 6, 397. https://www.doi.org.// 10.3389/fvets.2019.00397

Olatunde, G., \& Atungulu, G. (2018). Emerging petfood drying and storage strategies to maintain safety. Charter, 3, 45-61. Food and Feed Safety Systems and Analysis. http://dx.doi.org/10.1016/B978-0-12-811835-1.00003-8

Pearl, R., Wadden, T., Bach, C., Leonard, S., \& Michel, K. (2020). Who's a good boy? Effects of dog and owner body weight on veterinarian perceptions and treatment recommendations. International Journal of Obesity, 44(12), 2455 2464 https://doi.org/10.1038/s41366-020-0622-7

Ripps, H., \& Shen, W. (2012). Review: Taurine: A "very essential" amino acid. Molecular Vision, 18, $2673-2686$. http://www.molvis.org/molvis/v18/a275>

Roberts, M. T., Bermingham, E. N., Cave, N. J., Young, W., McKenzie, C. M., \& Thomas, D. G. (2018). Macronutrient intake of dogs, self-selecting diets varying in composition offered ad libitum. Journal of Animal Physiology and Animal Nutrition, 102,568-575. https://doi.org/10.1111/jpn.12794

RTC. (2012). Productos utilizados en alimentación animal y establecimiento. Pub. L. No. 65.05.52:11 http://www.pgrweb.go.cr/scij/Busqueda/Normativa/Normas/nrm_texto_completo.aspx?param1=NRTC\&nVal or1=1\&nValor2=73269\&nValor3=89862\&strTipM=TC

Salt, C., Morris P. J., Butterwick, R., Lund, R. F., Cole, T. J., \& German, A. J. (2020). Comparison of growth patterns in healthy dogs and dogs in abnormal body condition using growth standards. PLOS ONE, 15(9), e0238521, https://doi.org/ 10.1371/journal.pone.0238521

SEPSA (2014). Boletín Estadístico Agropecuario -№24. San José, Costa Rica: Secretaría Ejecutiva de Planificación Sectorial Agropecuaria Estudios(SEPSA).

Solís, C., \& Ramírez A. (2013). Tenencia y cuido de mascotas en las viviendas. En Madrigal, J. Encuesta Actualidades. Escuela de Estadística (pp. 38-41.). San José, Costa Rica: Universidad de Costa Rica. http://www.estadistica.ucr.ac.cr/index.php/es/encuestas/actualidades?filter\%5Bsearch\%5D=2013

Trat, Q. D., Hendriks, W. H., \& van der Poel, A. F. B. (2008). Effects of extrusion processing on nutrients in 
drypetfood. Journal of the Sciencie of Food and Agriculture, 88, 1487-1493.

Torda-Orsolya, J., Kata, V., Junó-Vanda, K., \& Pongrácz, P. (2020). Factor affecting canine obesity seem to be independent of the economic status of the country- A survey on Hungarian companion dogs. Animals, 10, 1267 https://doi.org/10.3390/ani10081267

van Herwijnen, I.R., Corbee, R.J., Endenburg, N.,Beerda, B. \& van der Borg, J.A.M. (2020) Permissive parenting of the dog associates with dog overweight in a survey among 2,303 Dutch dog owners. PLOS ONE, 15(8), e0237429. https://doi.org/10.1371/journal.pone.02374.29

WAP (Protección Animal Mundial). (2016). Estudio Nacional sobre tenencia de perros en Costa Rica. San José, Costa Rica. https://issuu.com/wspalatam/docs/estudioperros-web-singles

Ward, E. (2015). Las 5 preguntas más importantes sobre la obesidad de mascotas: cómo chequear, qué alimentar y cómo ejercitar. Asociación para la Prevención de Obesidad de Mascotas. Estados Unidos. https://petobesityprevention.org/news/2015/10/19/the-5-biggest-questions-you-must-ask-about-pet-obesityhow-to-check-what-to-feed-and-how-to-exercise

Wrap (2009). Efficient use of resource in pet food packaging design. Pet food report. Material change for a better environment. http://www.wrapni.org.uk/sites/files/wrap/RSC003-009\%20Pet\%20Food\%20Report1.pdf

Yam, P. S., Butowski, C. F., Chitty, J. L., Naughton, G., Wiseman-Orr, M. L., Parkin, T., \& Reid, J. (2016). Impact of canine overweight and obesity on health -related quality of life. Preventive Veterinary Medicine, 127, 64-69. https://doi.org/10.1016/j.prevetmed.2016.03.013

Zicker, S. (2008). Evaluating pet foods: How confident are you when you recommend a comercial pet food?. Topics in Companion Animal Medicine,23(3),121-126. https://doi.org/10.1053/j.tcam.2008.04.003 


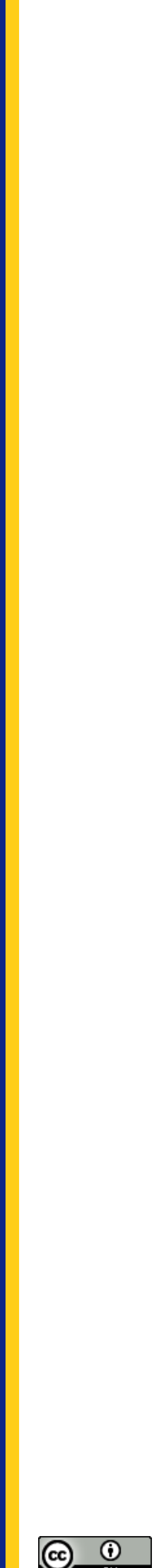

(c) (i) ${ }_{\text {oPen }}$ Access

UNED Research Journal (e-ISSN 1659-441X), Vol. 13(2): e3385, December, 2021 